\title{
ZEROS OF SUCCESSIVE DERIVATIVES OF ENTIRE FUNCTIONS
}

\author{
BY R. P. BOAS, JR. AND A. R. REDDY
}

Communicated by R. C. Buck, June 5, 1972

Let $f(z)$ be a transcendental entire function. If $r_{k}$ is the radius of the largest disk with center at 0 in which $f^{(k)}(z)$ is zero-free, it is known that, when $f(z)$ is of positive finite order $\rho$ and $\alpha>\rho$, there is an infinite increasing sequence of values of $k$ such that $r_{k} \geqq k^{(1 / \alpha)-1}(\AA$ lander [1] for $\rho<1$; stated by Pólya [4] for $\rho>1$ also; the first published proof for $\rho>1$ was given by Erdös and Rényi [3], where Ålander's result is misquoted as being for $\rho>1$ ). When $\rho=1$ and $f(z)$ is of exponential type $\tau$ it is known more precisely that $r_{k} \geqq c(\tau)$ (Takenaka [5]; for modern results see Buckholtz and Frank [2]).

We have established the existence of larger zero-free disks if they are no longer required to be centered at 0 . Our principal results are as follows.

THEOREM 1. If $f(z)$ is an entire function at most of order 2, finite type, there is an arbitrarily large disk, somewhere in the plane, in which an infinity of $f^{(k)}(z)$ are zero-free.

This is a corollary of Ålander's theorem for $\rho<1$, but not for $1 \leqq \rho \leqq 2$.

The conclusion of Theorem 1 fails for entire functions of order greater than 2 .

THEOREM 2. If $\rho>2$, there is an entire function of order $\rho$ such that, for some positive A, every disk, anywhere in the plane, of radius $A$ contains a zero of every $f^{(k)}(z)$.

THEOREM 3. If $f(z)$ is an entire function of finite order $\rho \geqq 2$, and $\alpha>\rho$, there is a point $z_{0}$ such that, for an infinity of $k$, we have $f^{(k)}(z) \neq 0$ in $\left|z-z_{0}\right|<k^{(1 / \alpha)-1 / 2}$.

Theorem 3 shows that when we do not require the concentric zero-free disks to be centered at a prescribed point, they can be appreciably larger than in Pólya's theorem.

THEOREM 4. If $f(z)$ is an entire function, for every (arbitrarily large) $c>0$, there is a $z_{0}$ such that $f^{(k)}(z) \neq 0$ in $\left|z-z_{0}\right|<c k^{-1 / 2}$ for an infinity of $k$.

THEOREM 5. If $f(z)$ is analytic in $|z|<R$, there are a (possibly small) $c>0$ and a point $z_{0}$ in $|z|<R$ such that $f^{(k)}(z) \neq 0$ in $\left|z-z_{0}\right|<c k^{-1 / 2}$ for an infinity of $k$.

AMS (MOS) subject classifications (1970). Primary 30A64, 30A66; Secondary 30A08. 
Results of this character are not altogether unexpected. For example, if $f(z)$ is of order $\rho$, so is each of its derivatives. Consequently each $f^{(k)}(z)$ has at most $O\left(R^{\rho+\varepsilon}\right)$ zeros in a disk of radius $R$; when $\rho<2$ this means that, for each $k$, if $R$ is large enough, $|z|<R$ contains an arbitrarily large disk in which $f^{(k)}(z) \neq 0$. In Theorem 1 , however, we have a single disk that is zero-free for each of an infinity of derivatives; and in Theorems 3-5 we have a sequence of concentric disks, of diminishing radii, such that, for a subsequence, each disk is zero-free for the corresponding derivative. To establish such results we require estimates, more precise than those used by Erdös and Rényi, for the number of zeros of $f^{(k)}(z)$ is a disk of prescribed radius.

It is interesting that the dividing line between "small order" and "large order" in this work is at order 2, rather than at order 1 as in the work of Alander and Pólya; particularly since there are other indications (Pólya [4]) that the zeros of successive derivatives tend to become scattered for order less than 2 and to become concentrated for order greater than 2 .

\section{REFERENCES}

1. M. Ålander, Sur le déplacement des zéros des fonctions entières par leur dérivation, Uppsala Thesis, 1914.

2. J. D. Buckholtz and J. L. Frank, Whittaker constants, Proc. London Math. Soc. (3) 23 (1971), 348-370.

3. P. Erdös and A. Rényi, On the number of zeros of successive derivatives of analvtic functions, Acta Math. Acad. Sci. Hungar. 7 (1956), 125-144. MR 18, 201.

4. G. Pólya, On the zeros of the derivatives of a function and its analytic character, Bull. Amer. Math. Soc. 49 (1943), 178-191. MR 4, 192.

5. S. Takenaka, On the expansion of integral transcendental functions in generalized Taylor's series, Proc. Physico-Math. Soc. Japan 14 (1932), 529-542.

Department of Mathematics, Northwestern University, Evanston, Illinois 60201 (current address of R. P. Boas, Jr.)

Department of Mathematics, University of Toledo, Toledo, Ohio 43606 (current address of A. R. Reddy)

Department of Mathematics, University of Missouri, St. Louis, Missouri 63121 